Images du travail, travail des images

5 | 2018

Le travail à l'écran : mise en scène des groupes professionnels par les médias

\title{
Le travail à l'écran. Mise en scène des groupes professionnels par les médias
}

Christian Papinot et Marc Perrenoud

\section{OpenEdition}

\section{Journals}

Édition électronique

URL : http://journals.openedition.org/itti/797

DOI : 10.4000/itti.797

\section{Éditeur}

Université de Poitiers

\section{Référence électronique}

Christian Papinot et Marc Perrenoud, «Le travail à l'écran. Mise en scène des groupes professionnels par les médias », Images du travail, travail des images [En ligne], 5 | 2018, mis en ligne le 01 février 2018, consulté le 14 avril 2021. URL : http://journals.openedition.org/itti/797 ; DOI : https://doi.org/10.4000/ itti.797

Ce document a été généré automatiquement le 14 avril 2021

Images du travail, travail des images 


\title{
Le travail à l'écran. Mise en scène des groupes professionnels par les médias
}

\author{
Christian Papinot et Marc Perrenoud
}

1 Le dossier de ce numéro d'Images du Travail, Travail des Images est consacré au travail et à la mise en scène des groupes professionnels lorsqu'ils sont portés à l'écran. Les articles qu'il rassemble sont issus de certaines communications présentées à l'occasion du colloque Montrer le travail et les groupes professionnels qui s'est tenu à l'Université de Lausanne en septembre 2015 et a été co-organisé par le Comité de Recherche 32 («Savoirs, métiers et identités professionnelles») de l'Association internationale des sociologues de langue française (AISLF), le GRESCO et le LACCUS.

2 Si l'on considère qu'un groupe professionnel est un « ensemble de travailleurs exerçant une activité ayant le même nom, dotés d'une visibilité sociale, bénéficiant d'une identification et d'une reconnaissance, occupant une place différenciée dans la division sociale du travail, et caractérisés par une légitimité symbolique » (Demazière et Gadéa (dir.), 2009, 20), le processus de construction de légitimité symbolique et d'autonomie professionnelle procède de logiques de visibilité sociale, d'identification, de reconnaissance, dans lesquelles les images matérielles des groupes professionnels peuvent être diversement mobilisées et mises en scène.

Dans le premier numéro de la revue, nous avions mis l'accent sur le fait que ces logiques de visibilité sociale relevaient d'abord d'une capacité des groupes professionnels à se mobiliser, à s'auto-organiser en développant diverses formes de représentation de soi réalisées ou commanditées, soit par des professionnels seuls, soit par des groupements de gens de métier. L'histoire des groupes professionnels apparaît ainsi jalonnée d'images matérielles, planes ou en relief, fixes ou animées, qui témoignent de la manière dont ils se voient eux-mêmes ou souhaitent être vus. Que disent les images des groupes professionnels? Dans quels contextes et avec quelles intentions sont-elles produites? Que révèlent-elles de la place occupée par les métiers dans le monde social dont ils font partie, de leur position dans la division du travail et 
dans les échelles de revenu, de pouvoir ou de prestige? Comment montrent-elles les activités de travail et les pratiques professionnelles? On a pu étudier ainsi la manière dont les groupes professionnels se mettaient eux-mêmes en scène, comment ils se donnaient à voir comme les personnages centraux du «drame social du travail », selon la formule d'Everett C. Hughes (1996), leurs membres étant engagés dans leur rôle avec leur tenue, leur gestuelle, leurs outils-accessoires, bref, leurs différents attributs. Si ces images peuvent obéir à des intentions diverses, par exemple une fonction technique (signalisation d'un chantier montrant une silhouette d'ouvrier) ou une valorisation individuelle ou collective (publicité dans la presse pour l'armée de terre ou les chambres notariales), elles participent aussi de ces stratégies plus ou moins délibérées de visibilité sociale contribuant à instituer un « idiome figuratif » de chaque métier et groupe professionnel (Gadéa, 2016).

Mais cette visibilité sociale des groupes professionnels n'est bien sûr pas produite de manière unilatérale et autonome. Si les groupes professionnels contrôlent en partie les représentations d'eux-mêmes et de leur activité, ce n'est évidemment que de façon partielle comme on le verra dans ce dossier thématique.

5 En écho donc au premier numéro d'Images du Travail, Travail des Images qui avait pour objet d'explorer les enjeux autour des stratégies de mise en images des groupes professionnels par eux-mêmes, le présent dossier est majoritairement consacré aux représentations audiovisuelles de l'activité laborieuse et/ou professionnelle produites par autrui et diffusées sur écran de télévision, de cinéma ou d'ordinateur.

6 Considérant avec Guy Debord que «le spectacle n'est pas un ensemble d'images, mais un rapport social entre des personnes médiatisé par cet ensemble d'images » (Debord, 1967), nous avons souhaité rassembler des contributions concernant le spectacle du travail abordé sous l'angle des métiers et des groupes professionnels, et appréhendé dans une optique pluridisciplinaire. Nous avons considéré le rôle déterminant de ces images du travail et des groupes professionnels en ce qu'elles participent massivement à la production de métaphores sur chacune des familles de métiers et plus globalement sur le travail contemporain et ses évolutions. Ces images matérielles sont d'autant plus importantes à étudier qu'elles envahissent notre quotidien dans lequel les écrans sont plus que jamais omniprésents et qu'elles permettent d'éclairer les processus de construction du monde social par l'«action symbolique», selon l'expression de Kenneth Burke (1966).

7 Cinéma documentaire, séries télévisées ou spectacles de télé-réalité, les images sur le travail et les groupes professionnels semblent toujours receler une dimension d'exemplarité à destination du public, appelant une réaction d'identification, d'admiration, ou de solidarité envers des travailleurs (voire des groupes professionnels) représentés en héros ou victimes, ou au contraire suscitant un rejet, par exemple du "mauvais travailleur" (voire de tout un groupe professionnel), ou encore une réprobation du type de rapports sociaux mis en spectacle (le "sale boulot» de l'huissier par exemple) comme du mépris de classe, à l'instar par exemple des images traditionnelles des « cris de Paris » (Milliot, 1995). Depuis les représentations anciennes jusqu'aux formes les plus contemporaines du récit par l'image animée, transmises par l'écran du cinéma, de la télévision ou de l'ordinateur (et ses avatars les plus mobiles), le travail et les groupes professionnels sont donnés à voir, souvent dans un but d'édification du spectateur. C'est ainsi son caractère normatif qui fonde le spectacle $d u$ travail, renforçant la dimension morale de l'activité laborieuse imprégnée de l'esprit du 
capitalisme (Weber, 2004; Kalinowsky, 2005) sous les apparences indolores du divertissement ou de l'information.

Image 1. Alphabet grotesque des cris de Paris, 1861

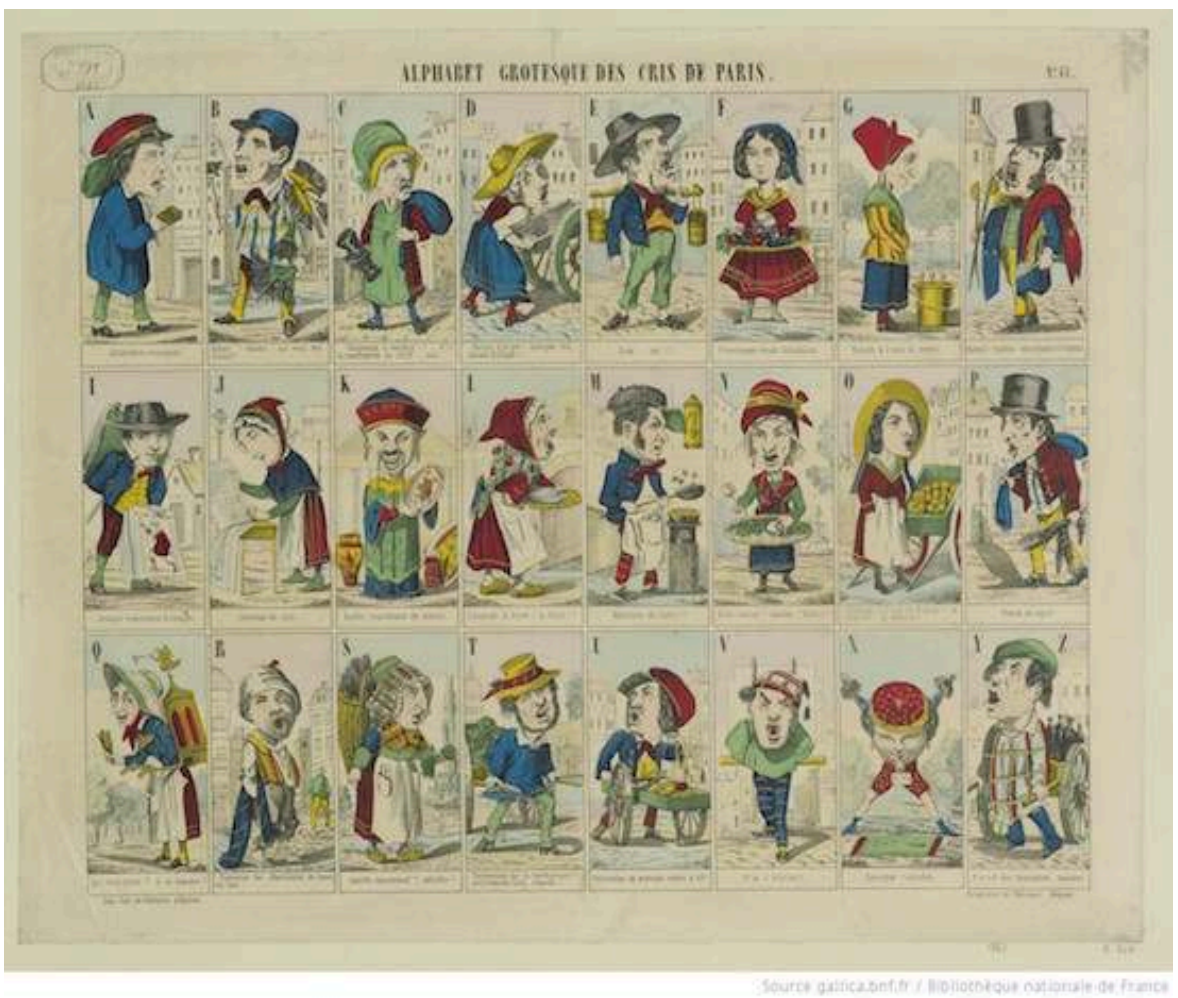

(c) Bibliothèque nationale de France, département Estampes et photographie, FOL-LI-59 (7)

Parler d'images véhiculées par les médias contemporains confronte d'emblée le chercheur à des matériaux peu aisés à analyser afin d'éviter les risques inhérents à toute analyse d'images, c'est-à-dire les risques multiples de la décontextualisation, de l'ethnocentrisme ou de l'anachronisme. Une image - a fortiori indiciaire - est une représentation conventionnelle et codée de la réalité, et donc d'abord et avant tout, une production culturellement déterminée qui obéit à des manières de montrer très codifiées socialement (Meyer et Papinot, 2016). Quelle cohérence par exemple pour le corpus iconographique de l'historien qui, en traversant les âges, nous donne à voir ensemble des images qui ont été jugées dignes d'être jalousement conservées et d'autres qui ont miraculeusement survécu aux autodafés iconoclastes? Comment analyser dans le même corpus iconographique des images rares, diffusées quasi clandestinement, avec des séries, tirées en grand nombre, archi-diffusées ? Et ensuite, comment analyser ces images qui ne sont évidemment pas toutes congruentes. Comment interpréter ces divergences de représentations d'un même groupe professionnel d'un support de diffusion à un autre? Comment analyser ces représentations divergentes produites dans le temps ou sur différents supports : images cinématographiques, télévisuelles, de presse écrite, etc ? Ou encore comment analyser les images sans perdre de vue les logiques de la réalisation, de la production plus globale dans laquelle elle prend place et sens? Ces questions transversales et bien d'autres s'imposent à qui souhaite analyser les groupes professionnels par les images qui sont en produites et elles traversent ce dossier d'un texte à l'autre. 


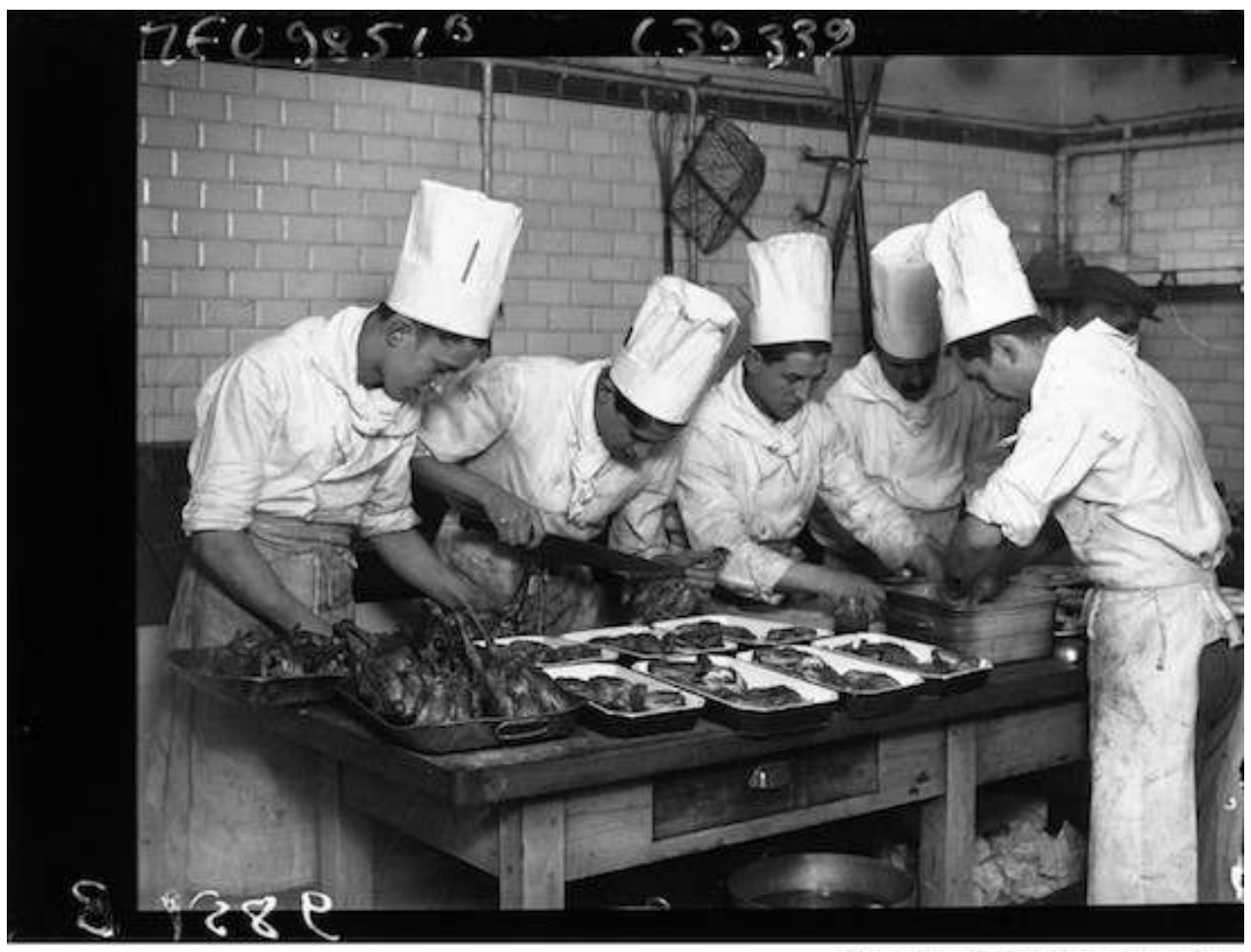

(C) Agence Meurisse, Bibliothèque nationale de France, département Estampes et photographie, El-13 (2915)

Dans le présent numéro thématique, on trouvera d'abord trois articles consacrés à des séries télévisées, depuis Les Atomistes des années 1960 jusqu'à la télé-réalité la plus contemporaine. Ainsi, Pascal Césaro et Pierre Fournier se sont-ils intéressés à une série télévisée datant de l'âge d'or de l'ORTF et présentant le travail d'une équipe d'ingénieurs atomistes dans la France des années 1960, au moment où se déploie le programme nucléaire dans notre pays au nom de l'indépendance nationale ( Se concentrer sur le travail pour mettre en feuilleton le monde nucléaire dans les années 1960 : opération de télévision-réalité ou de propagande?»). Si la télévision française court le risque de situer un feuilleton romanesque dans l'univers nucléaire, se demandent les auteurs, n'est-ce qu'avec un projet esthético-didactique, ou bien aussi pour répondre à des attentes différentes qui feraient des Atomistes un feuilleton à part? La contextualisation historique opérée vient démontrer que la série a bien servi la volonté de l'État de convaincre l'opinion publique de l'importance du développement civil aussi bien que militaire de l'industrie nucléaire. Concernant l'époque contemporaine, la série télévisée Ainsi soient-ils étudiée par Benoît Verdier met en scène des futurs prêtres étudiant au séminaire, la diversité des profils de séminaristes et des situations dans lesquelles ils évoluent dans leur rapport au monde contemporain donnant l'occasion de montrer à la fois le travail de formation exercé par l'institution religieuse et les interactions entre les prêtres en formation (« La formation ecclésiale au prisme d'une série TV : Reformulation de l'idéal vocationnel »). Dès la première saison, les séminaristes sont montrés comme des jeunes de leur époque dans un format pédagogique semblable à celui de l'enseignement supérieur général, l'intentionnalité du réalisateur semblant être de dé-singulariser cette formation tout en réactivant 
l'idéal vocationnel sacerdotal. C'est à une autre déclinaison de l'idéologie individualiste de la vocation et de la morale que s'est intéressé Marc Perrenoud à travers l'étude de deux émissions dites de "télé-réalité " plaçant les situations de travail au coeur de la dramaturgie ( Pour une étude du travail et des métiers vus par la télé-réalité ») et notamment les métiers de cuisinier (Cauchemar en cuisine) et de chef d'entreprise (Patron incognito). Dans ces différentes configurations, on a affaire à l'euphémisation, voire à la dénégation des rapports sociaux à l'œuvre dans un restaurant et dans la plupart des situations de travail (par exemple l'impossible adéquation des intérêts respectifs des clients, des salariés et du patron) au profit d'une idéologie individualiste de la vocation et de la morale, parfaitement en accord avec l'esprit du capitalisme.

On trouvera ensuite deux textes qui portent sur les représentations des groupes professionnels à l'écran cinématographique et de l'ordinateur. Il s'agit d'abord d'un essai de Jean-Paul Géhin sur la représentation d'un métier dans le cinéma documentaire à partir de l'exemple du métier d'ouvrier d'abattoir («Comment le cinéma documentaire de création montre-t-il les métiers? L'exemple des ouvriers d'abattoirs ») qui montre que ces films au fond montrent peu le travail au profit de ce que l'auteur appelle le "systématisme industriel » qui organise la transformation de l'animal vivant en bien de consommation et focalise l'attention sur deux dimensions saillantes : la violence faite aux animaux et la fascination exercée par cette industrie de flux avec son cortège d'accumulation de carcasses, de gestes répétitifs et de cadences accélérées. L'article de Samuel Chagnard, enfin, traite la question de façon plus adjacente et fait le lien en cela avec le premier numéro de la revue puisqu'il étudie les vidéos produites par l'Orchestre national de Paris pour présenter sur son site internet les différents instruments de l'orchestre, présentation assurée par des instrumentistes dont l'auteur montre combien ils sont littéralement identifiés à leurs instruments. L'analyse du dispositif audiovisuel de présentation des instruments permet de rendre compte des processus conjoints de personnification de l'instrument et d'identification à l'instrument qui transforment l'instrumentiste en "instrumentiste-instrument " («Dedans, dehors: la place de l'instrumentiste-instrument d'orchestre symphonique »).

Gageons qu'à la lecture de ce dossier croisant les perspectives de la sociologie du travail, de la sociologie de l'image et des médias ainsi que des sciences de l'information et de la communication, on comprendra tout l'intérêt d'une étude aussi systématique que possible du spectacle du travail et des métiers dans les médias de masse.

\section{BIBLIOGRAPHIE}

Burke K., (1966), Language as Symbolic Action: Essays on Life, Literature, and Method, Berkley, U. of California Press.

Debord G., (1967), La société du spectacle, Paris, Buchet/Chastel.

Demazière D. et Gadéa C. (dir.), 2009, Sociologie des groupes professionnels, Paris, La Découverte. 
Gadea C., (2016), « L'idiome figuratif des groupes professionnels », Images du travail, Travail des images, $n^{\circ} 1$. [En ligne] 1-2016, mis en ligne le 28 juin 2016, consulté le 1 juillet 2016. URL : http:// imagesdutravail.edel.univ-poitiers.fr/index.php?id=377

Hughes E. C., (1996), Le regard sociologique, Paris, EHESS.

Kalinowski I., (2005), « Le capitalisme et son “éthique”, une lecture de Max Weber », Agone, $\mathrm{n}^{\circ}$ 33, p. 253-264.

Meyer M. et Papinot C. (dir.), 2016, « Le travail des images dans la démarche de recherche. Analyse réflexive et compréhension de l'objet ", Images du travail Travail des images, $n^{\circ} 3$. [En ligne] 3-2016, mis en ligne le 15 décembre 2016, consulté le 11 février 2017. URL : http:// imagesdutravail.edel.univ-poitiers.fr/index.php?id=1120

Milliot V., (1995), Les cris de Paris ou le peuple travesti, Paris, Publications de la Sorbonne.

Weber M., (2004) [1904], L'éthique protestante et l'esprit du capitalisme, Paris, Gallimard.

\section{AUTEURS}

\section{CHRISTIAN PAPINOT}

Université de Poitiers - GRESCO

MARC PERRENOUD

Université de Lausanne - LACCUS 